\title{
Pion production in neutrino interactions with nuclei
}

\author{
T. Leitner*, O. Lalakulich*, O. Buss*, U. Mosel* and L. Alvarez-Ruso ${ }^{\dagger}$ \\ * Institut für Theoretische Physik, Universität Giessen, Germany \\ ${ }^{\dagger}$ Departamento de Física, Centro de Física Computacional, Universidade de Coimbra, Portugal
}

\begin{abstract}
Neutrino-induced pion production on nuclear targets is the major inelastic channel in all present-day neutrinooscillation experiments. It has to be understood quantitatively in order to be able to reconstruct the neutrino-energy at experiments such as MiniBooNE or K2K and T2K. We report here results of cross section calculations for both this channel and for quasielastic scattering within the semiclassical GiBUU method. This methods contains scattering, both elastic and inelastic, absorption and side-feeding of channels all in a unitary, common theoretical framework and code. We find that charged current quasielastic scattering (CCQE) and $1 \pi$ production are closely entangled in actual experiments, due to final state interactions of the scattered nucleons on one hand and of the $\Delta$ resonances and pions, on the other hand. We discuss the uncertainties in the elementary pion production cross sections from ANL and BNL. We find the surprising result that the recent $1 \pi$ production cross section data from MiniBooNE are well described by calculations without any FSI. For higher energies we study the validity of the Bloom-Gilman quark-hadron duality for both electron- and neutrino-induced reactions. While this duality holds quite well for nucleon targets, for nuclear targets the average resonance contributions to the structure function $F_{2}$ are always lower than the DIS values. This result indicates a significant impact of nuclear effects on observables, reducing the cross section and structure functions by at least $30-40 \%$ and changing the form of various distributions.
\end{abstract}

Keywords: neutrino-nucleus interactions, pion production, quasielastic scattering

PACS: $13.15 .+\mathrm{g}, 25.30 . \mathrm{Pt}$

\section{INTRODUCTION}

Neutrino oscillations experiments search for a distortion in the neutrino flux at the detector positioned far away from the source. By comparing both near and far neutrino energy spectra, one gains information about the oscillation probability and with that about mixing angles and mass squared differences. However, the neutrino energy, that enters critically into the oscillation probability, is not directly measurable but has to be reconstructed from the reaction products. A proper understanding of neutrino-nucleus interactions is, therefore, essential for the interpretation of current neutrino oscillations experiments. Present $v_{\mu}$ disappearance experiments use the CCQE reaction both as signal event and to reconstruct the neutrino energy from the outgoing muon. CCQE is defined as $v_{\ell} n \rightarrow \ell^{-} p$ on a single nucleon; in the nucleus, CCQE is masked by final-state interactions (FSI). Thus, the correct identification of CCQE events is directly related to the question of how FSI influence the event selection. The main background to CCQE is $\mathrm{CC} 1 \pi^{+}$production. If the pion is absorbed in the nucleus and/or not seen in the detector, these events can be misidentified as CCQE. Consequently, a proper understanding of CCQE and $\mathrm{CC} 1 \pi^{+}$is essential for the reconstruction of the neutrino energy.

The main task in a $v_{e}$ appearance experiment like MiniBooNE is to detect electron neutrinos in a (almost) pure $v_{\mu}$ beam. The signal event, the $v_{e}$ CCQE interaction, is dominated by background. A major problem comes from misidentified events, mainly because of the fact, that the MiniBooNE detector cannot distinguish between a photon and an electron. Thus, $v_{\mu}$ induced neutral current $\pi^{0}$ production, where the $\pi^{0}$ decays into two $\gamma_{\mathrm{s}}$, is the major source of background when one of the photons is not seen or both Cherenkov rings overlap.

As all of the present oscillations experiments use nuclear targets, it is mandatory to consider FSI, i.e., pion rescattering, with and without charge exchange, and absorption in the nuclear medium. A realistic treatment of the final state interactions (FSI) can be achieved in the framework of a coupled-channel transport theory - the GiBUU model.

After a brief review of our model, we first discuss the impact of pion production on CCQE measurements. We further investigate the influence of nuclear effects on $\mathrm{CC} 1 \pi^{+}$and $\mathrm{NC} 1 \pi^{0}$ cross sections, and, where possible, we confront our model to recent data measured at MiniBooNE. In the second part we extend the description to higher energies using duality arguments.

\section{GIBUU MODEL}

We treat neutrino-nucleus scattering as a two-step process. In the initial-state step, the neutrinos interact with nucleons embedded in the nuclear medium. In the finalstate step, the outgoing particles of the initial reaction are propagated through the nucleus using a hadronic transport approach. 


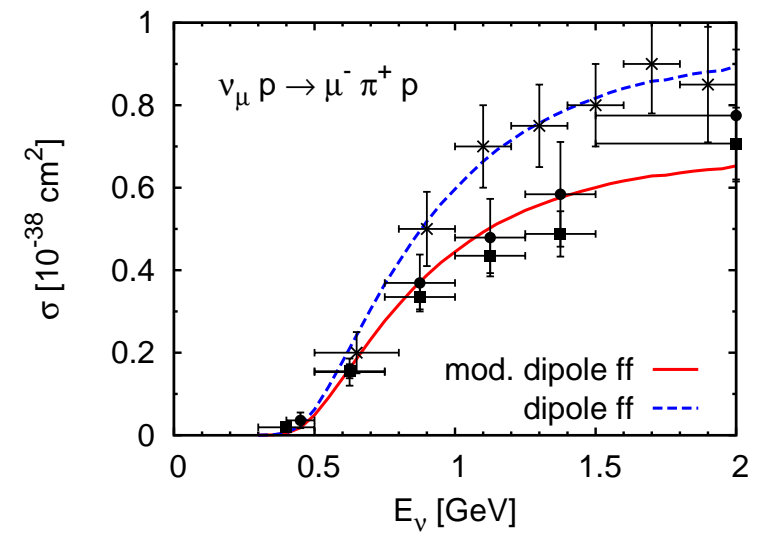

FIGURE 1. Total $v_{\mu} p \rightarrow \mu^{-} \pi^{+} p$ cross section as a function of the neutrino energy compared to the pion production data of of ANL (Refs. [5] (•), [6] (ם)) and BNL ([7] (×)). The solid line has been obtained with a form factor fitted to the ANL data, the dashed one is fitted to the BNL data.

In the energy region relevant for MiniBooNE, SciBooNE and $\mathrm{K} 2 \mathrm{~K}$, the elementary $v N$ reaction is dominated by two processes: quasielastic scattering and the excitation of the $\Delta$ resonance $\left(\mathrm{P}_{33}(1232)\right)$. Additionally, our model includes the excitation of $12 N^{*}$ and $\Delta$ resonances with invariant masses less than $2 \mathrm{GeV}$ and also a non-resonant single-pion background. Details are given in [1].

The excitation of the resonances ( $\mathrm{R})$ is described within the isobar model with the help of the nucleon, $N-\Delta$ and $N-N^{*}$ form factors. Vector form factors are derived from MAID helicity amplitudes [2, 3, 4] extracted from electron scattering experiments.

Experimental information on the N-R axial form factors is very limited. Goldberger-Treiman relations have been derived for the axial couplings, but there is no information about the $Q^{2}$ dependence. We apply PCAC and pion pole dominance to derive one of the axial couplings for each resonance and to relate it to the pseudoscalar coupling. The $Q^{2}$ dependence of the $\Delta$ axial form factors are fitted to either ANL or BNL bubble chamber neutrino-scattering $d \sigma / d Q^{2}$ data for the $v_{\mu} p \rightarrow \mu^{-} \pi^{+} p$ reaction. Fig. 1 shows the integrated cross section together with the data. We note already here, that the solid curve fits the ANL data, while the dashed curve fits the BNL data. Thus, the latter would obviously lead to higher pion production cross section also on the nucleus.

The single- $\pi$ non-resonant background cross section $\sigma_{\mathrm{BG}}$ includes vector, axial and also interference contributions

$$
\sigma_{B G}=\sigma_{\mathrm{BG}}^{\mathrm{V}}+\sigma_{\mathrm{BG}}^{\mathrm{A}}+\sigma_{\mathrm{BG}}^{\mathrm{V} / \mathrm{A}}=\sigma_{\mathrm{BG}}^{\mathrm{V}}+\sigma_{\mathrm{BG}}^{\mathrm{non}-\mathrm{V}} .
$$

The vector part is fully determined by electron scattering data, as described in [1]. The axial and the interference

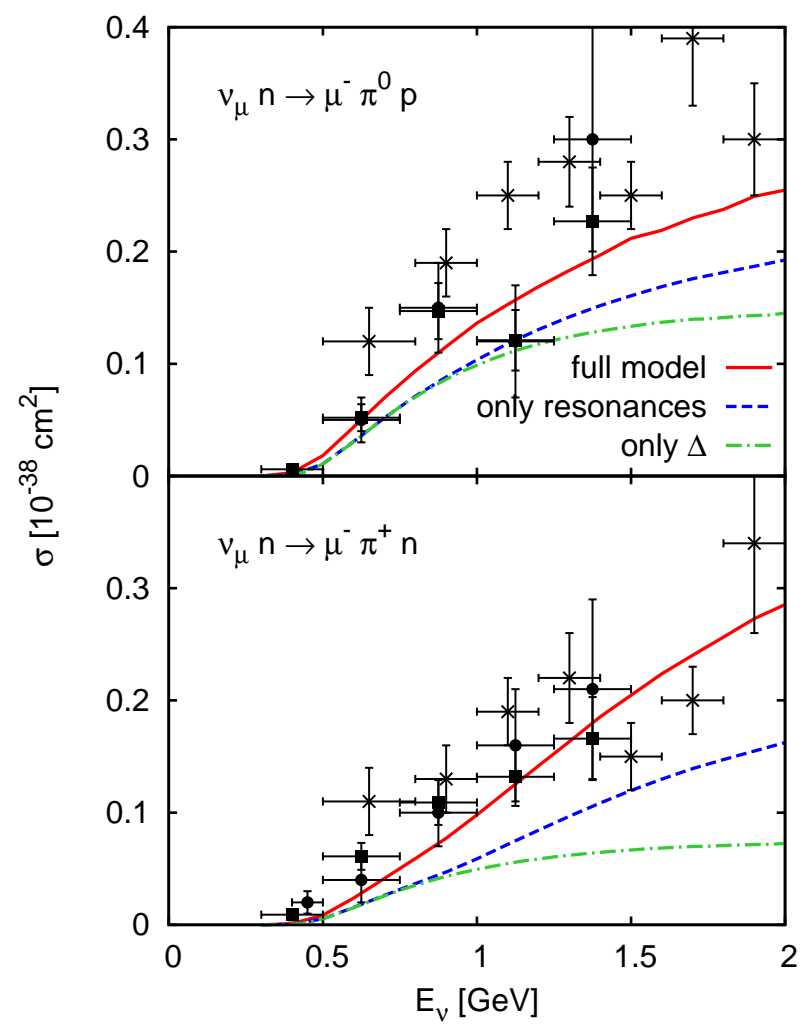

FIGURE 2. Total CC pion production cross sections for the mixed isospin channels as a function of the neutrino energy compared to the pion production data of of ANL (Refs. [5] $(\bullet)$ and [6] (ם)) and BNL $([7](\times))$. The solid lines denote the our full result including the non-resonant background. Furthermore, we show the results for pion production only through the excitation and the subsequent decay of all resonances (dashed lines) or through the $\Delta$ alone (dash-dotted lines). Note that the ANL based fit for the $\Delta$ axial form factor has been used here.

term collected under the label "non- $\mathrm{V}$ " are only present in neutrino scattering and are fitted to the available neutrino data for both, $v_{\mu} n \rightarrow \mu^{-} \pi^{+} n$ and $v_{\mu} n \rightarrow \mu^{-} \pi^{0} p$. The final results are shown in Fig. 2

The neutrino-nucleon cross sections are modified in the nuclear medium. Bound nucleons are treated within a local Thomas-Fermi approximation. They are exposed to a mean-field potential which depends on density and momentum. We account for this by evaluating the above cross sections with full in-medium kinematics, i.e., hadronic tensor and phase-space factors are evaluated with in-medium four-vectors. We also take Pauli blocking and collisional broadening of the outgoing hadrons into account. Our model for neutrino-(bound)nucleon scattering is described in detail in [1].

After the initial neutrino-nucleon interaction, the produced particles propagate through and out of the nucleus. During propagation they undergo FSI which are simulated with the coupled-channel semi-classical GiBUU 

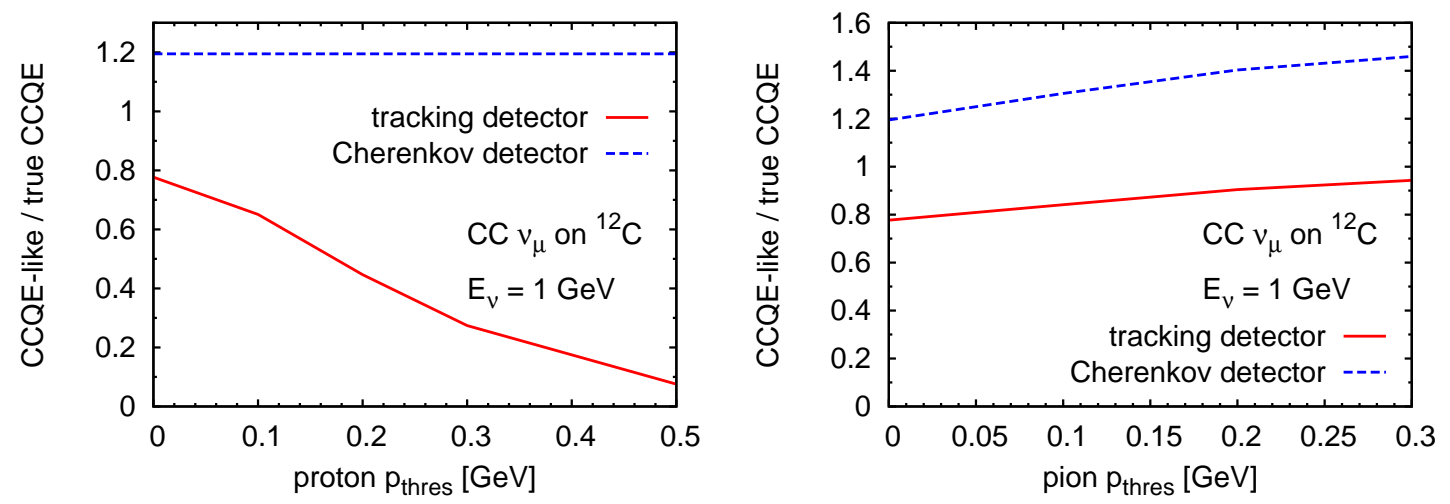

FIGURE 3. Ratio of the CCQE-like to the true CCQE cross section as a function of the proton (pion) momentum detection threshold for $\mathrm{CC} v_{\mu}$ on ${ }^{12} \mathrm{C}$ at $E_{v}=1 \mathrm{GeV}$. The solid lines are obtained using the tracking detector identification, while the dashed lines are for Cherenkov detectors.

transport model (for details, see Ref. [8] and references given there). It is based on the BUU equation which describes the space-time evolution of a manyparticle system in a mean-field potential including a collision term. Nucleons and resonances acquire mediummodified spectral functions and are propagated off-shell. Herby we ensure, that vacuum spectral functions are recovered after leaving the nucleus. The collision term accounts for changes (gain and loss) in the phase-space density due to elastic and inelastic collisions between the particles, and also to particle decays into other hadrons. Baryon-meson two-body interactions (e.g., $\pi N \rightarrow \pi N$ ) are described by resonance contributions and a small non-resonant background term; baryon-baryon cross sections (e.g., $N N \rightarrow N N, R N \rightarrow N N, R N \rightarrow R^{\prime} N, N N \rightarrow$ $\pi N N)$ are either fitted to data or calculated, e.g., in pion exchange models. The three-body channels $\pi N N \rightarrow N N$ and $\Delta N N \rightarrow N N N$ are also included. The BUU equations for all particle species are thus coupled through the collision term and also through the potentials. Such a coupled-channel treatment is required to account for side feeding into different channels.

The treatment of pion final state interactions in GiBUU has been widely tested both with $\pi A$ and $\gamma A \rightarrow$ $\pi X$ data $[9,10,11]$. The latter reaction is quite similar to the $v A \rightarrow \pi X$ reaction in that the incoming particle interacts with all target nucleons and the vector couplings are the same.

\section{CCQE AND CC $1 \pi^{+}$ENTANGLEMENT}

One challenge is to identify true CCQE events in the detector, i.e., muons originating from an initial QE process. The difficulty comes from the fact that the true CCQE events are masked by FSI in a detector built from nuclei. In general, at Cherenkov detectors such as MiniBooNE
CCQE-like events are all those where no pion is detected while in tracking detectors such as K2K-SciBar/SciFi CCQE-like events are those where a single proton track is visible and at the same time no pions are detected. In both detector types the FSI lead to misidentified events, e.g., an initial $\Delta$ whose decay pion is absorbed or which undergoes "pion-less decay" can count as CCQE event - we call this type of background events "fake CCQE" events. We denote every event which looks like a CCQE event by "CCQE-like".

To investigate the relation between the CCQE-like and true CCQE cross section, we show their ratio as a function of proton and pion momentum thresholds in Fig. 3 , As the proton is not at all relevant for the CCQE identification in Cherenkov detectors, the ratio is independent of the proton momentum detection threshold (dashed line in left panel). This is very different in tracking detectors which rely on the detected proton - here the efficiency is reduced to $\approx 10 \%$ at a proton momentum threshold of $0.5 \mathrm{GeV}$ (solid line in left panel). Even at $|\vec{p}|_{\text {thres }}^{p}=0$ the efficiency does not exceed $80 \%$ because of chargeexchange processes that lead to the emission of undetected neutrons and because of secondary proton knockout that leads to multiple-proton tracks. Focussing on the right panel of Fig. 3 we find that the CCQE-like cross section increases for both detector types as $|\vec{p}|_{\text {thres }}^{\pi}$ increases. In this case even more events with pions in the final state appear as CCQE-like because these pions are below threshold and thus not detected.

Fixing the flux normalization with HARP's pionproduction data, the MiniBooNE collaboration has presented their first, preliminary absolutely normalized total, differential and double differential cross sections for CCQE and finds an excess of about 35\% compared to the total cross section measured at NOMAD, ANL and BNL [12]. We emphasize that these absolute cross sections depend directly on the pion background subtraction 

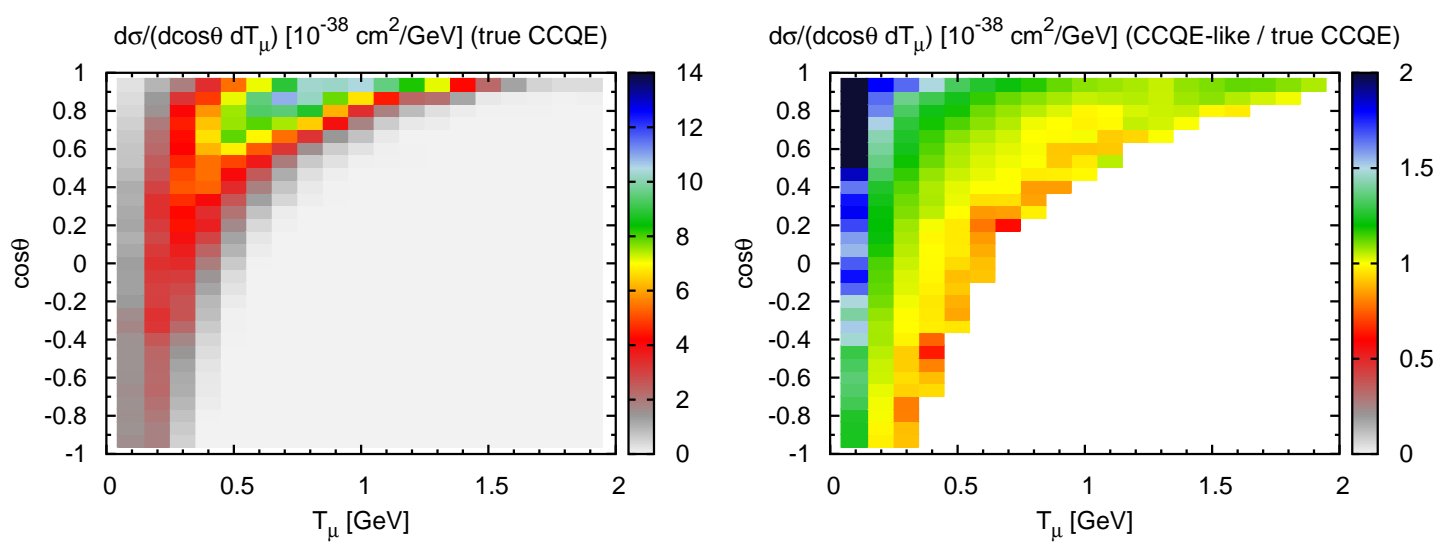

FIGURE 4. Double differential cross section on ${ }^{12} \mathrm{C}$ averaged over the MiniBooNE flux as a function of the muon kinetic energy and the muon scattering angle. The left panel shows the true CCQE cross section, the right panel the ratio of the CCQE-like to the true CCQE cross section.

which again is based on the Monte Carlo prediction (cf. Ref. [12]).

In Fig.4, we show our prediction for the double differential cross section at MiniBooNE in muon observables, all calculated with $M_{A}=1 \mathrm{GeV}$. The left panel shows the true CCQE events. To emphasize the role of "fake" CCQE events, we show the ratio CCQE-like/true CCQE in the right panel. This ratio shows that fakes contribute mainly at high energy transfers (low $T_{\mu}$ ) and forward angles. Unlike for monochromatic beams, the $\mathrm{QE}$ and $\Delta$ peaks are not distinguishable any more but strongly overlap. This fact makes a model-independent cut based on muon variables to subtract the background impossible.

\section{MINIBOONE'S CC $1 \pi^{+}$MEASUREMENT}

In Fig. [5 we give our results for the single- $\pi^{+} / \mathrm{QE}$ ratio for $\mathrm{CC}$ interactions on mineral oil $\mathrm{CH}_{2}$. The solid lines denote the $\mathrm{CC} 1 \pi^{+}$-like/CCQE-like result, the dashed lines stand for the true $\mathrm{CC} 1 \pi^{+}$/true CCQE result, and the dash-dotted lines give the vacuum expectation. Note that we have applied the Cherenkov detector identification criteria.

We emphasize that nuclear corrections cancel out in the ratio, only as long as FSI are not considered ("true" vs. "free"). In general, the complexity of FSI prevent such cancellations as one can infer from the result denoted with "like" which does not coincide with the "true" and "free" ones.

We further compare to very recent MiniBooNE data [13] (Fig. 5). Let us first focus on the data denoted with the triangles (upper data set). These are corrected for FSI using a specific Monte Carlo generator, i.e., they give the cross sections for bound nucleons "before FSI". As this procedure introduces a model dependence in the data, a

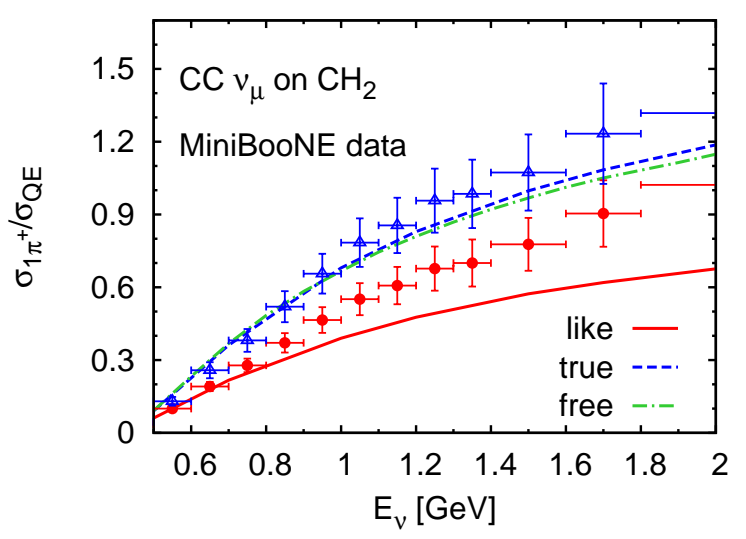

FIGURE 5. Single- $\pi^{+} / \mathrm{QE}$ cross section ratio for $\mathrm{CC}$ interactions vs. neutrino energy on $\mathrm{CH}_{2}$ together with recent data from MiniBooNE [13] (upper data set: corrected for FSI, lower data set: uncorrected for FSI). The solid lines denote the $\mathrm{CC} 1 \pi^{+}$-like/CCQE-like result (Cherenkov detector definitions), the dashed lines stand for the true $\mathrm{CC} 1 \pi^{+} /$true CCQE result, and the dash-dotted lines give the vacuum expectation, i.e., the sum of the nucleon cross sections (with two additional protons in the MiniBooNE case).

fully consistent comparison is not possible. Ignoring this inconsistency, our calculation denoted with "true" should be the one to compare with. The agreement is perfect for energies up to $1.5 \mathrm{GeV}$, and still within their error bars for higher $E_{v}$. The MiniBooNE data denoted with bullets (lower data set) is their result for the ratio of $\mathrm{CC} 1 \pi^{+}$-like to CCQE-like. As these data are not corrected for FSI within a specific Monte Carlo event generator, this observable is less model dependent. Still, the energy reconstruction requires specific assumptions as well as the detector simulation. We find that our calculation clearly underestimates the uncorrected data. However, the perfect 

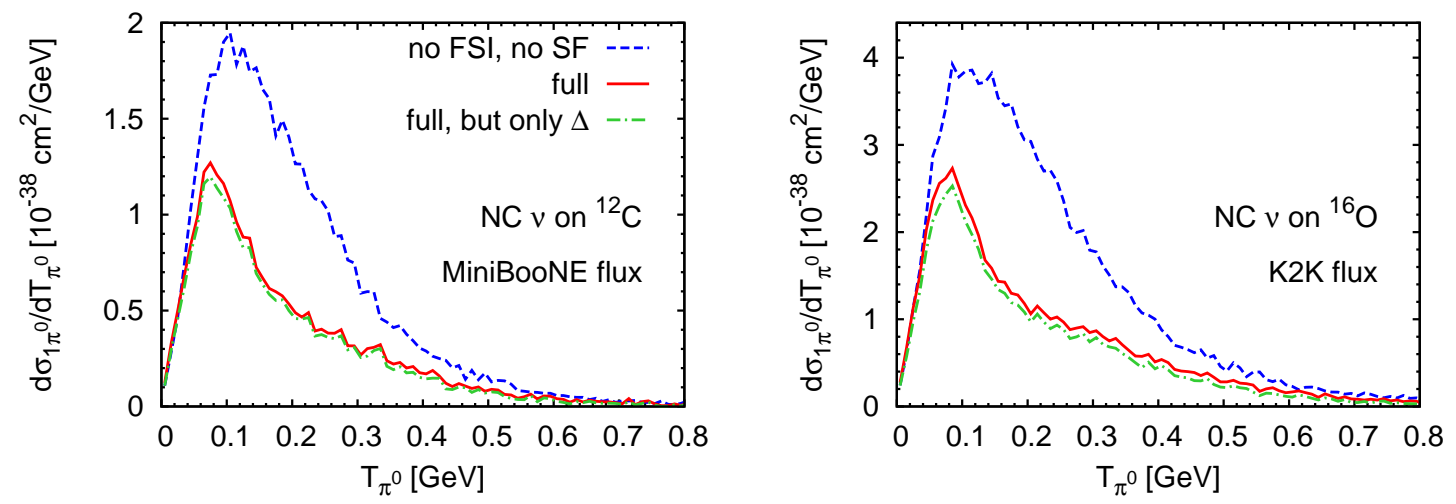

FIGURE 6. Left panel: NC induced single- $\pi^{0}$ production on ${ }^{12} \mathrm{C}$ as a function of the pion kinetic energy averaged over the MiniBooNE flux. Right panel: same on ${ }^{16} \mathrm{O}$ averaged over the $\mathrm{K} 2 \mathrm{~K}$ flux. The dashed lines show our calculation without FSI or spectral functions, both included in the full calculation denoted with the solid lines. The dash-dotted lines indicate the contribution from the $\Delta$ resonance to the full calculation.

agreement with their corrected distribution indicates a significant difference between the pion absorption models.

The underestimate of the pion/quasielastic ratio in particular at higher energies could be due to, among other possibilities, an underestimate of the pion production cross section or an overestimate of the CCQE-like cross section. Both depend directly on the input at the nucleon level, i.e., in particular on the axial form factor $C_{5}^{A}$ of the $\Delta$ resonance (see Fig. 11). For the results presented here, we have used the ANL data as a reference. However, we have shown in Ref. [14] that an increase of the total pion production cross section on the nucleon compatible with the BNL data also seems to be insufficient to describe this ratio at all energies. We note that a similar result for the ratio has been recently obtained by Athar et al. [15].

\section{$\mathrm{NC} 1 \pi^{0}$}

In the left panel of Fig. 6, we show our results for NC single- $\pi^{0}$ production off ${ }^{12} \mathrm{C}$ as a function of the pion kinetic energy. We have averaged over the MiniBooNE energy flux which peaks at about $0.7 \mathrm{GeV}$ neutrino energy [16]. In NC reactions the total pion yield is dominated by $\pi^{0}$ production, while $\pi^{+}$dominate in CC processes (for details, see Ref. [17] and references therein). Comparing the dashed with the solid line (results without FSI and spectral function vs. full calculation), one finds a considerable change. The shape is caused by the energy dependence of the pion absorption and rescattering cross sections. Pions are mainly absorbed via the $\Delta$ resonance, i.e, through $\pi N \rightarrow \Delta$ followed by $\Delta N \rightarrow N N$. This explains the reduction in the region around $T_{\pi}=0.1-0.3 \mathrm{GeV}$. Pion elastic scattering $\pi N \rightarrow \pi N$ reshuffles the pions to lower momenta and leads also to charge exchange scat-

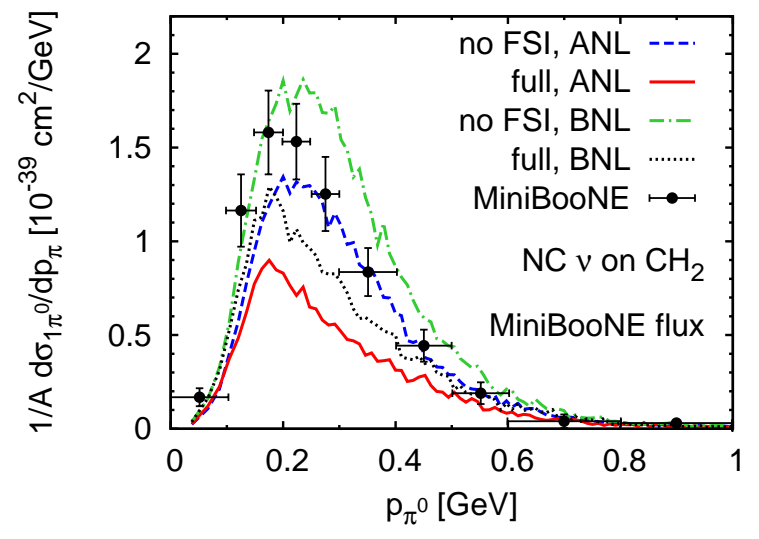

FIGURE 7. $\mathrm{NC}$ induced single- $\pi^{0}$ production on $\mathrm{CH}_{2}$ as a function of the pion momentum averaged over the MiniBooNE flux. The dashed and the solid lines denote the calculation with a modified dipole form factor (fitted to ANL data) for the $\Delta$ resonance (identical to the result in the left panel of Fig. 6). The dash-dotted and dotted lines are obtained with a dipole form for the form factor (fitted to the BNL data). The MiniBooNE data are taken from Ref. [18].

tering into the charged pion channels. The vast majority of the pions comes from initial $\Delta$ excitation (dash-dotted line), their production in the rescattering of nucleons is not significant at these energies.

The right panel of Fig. 6 shows the results for $\mathrm{NC}$ single- $\pi^{0}$ production off ${ }^{16} \mathrm{O}$ averaged over the K2K energy flux which peaks at about $1.2 \mathrm{GeV}$ neutrino energy [19]. Compared to the left panel, the spectrum is broader and extends to larger $T_{\pi}$ due to the higher neutrino energy. Again, pion production through initial QE scattering is not sizable.

In Fig. 7 we show also the latest $\mathrm{NC} \pi^{0}$ differential 
cross sections from MiniBooNE [18]. It can be seen that the full line, depicting the results of our calculation after FSI with the fit of the elementary cross section to the ANL data lies nearly a factor 2 below the data. The dashed line gives the result of the same calculation before FSI, the dash-dotted line shows the same quantity, but using a fit to the BNL data for the elementary cross section. We find it remarkable that these two curves (without FSI) are comparable to the data (with FSI), even though pion FSI have a major effect on the spectra (see Fig. 6). The absolute height of the experimental cross section is thus hard to reconcile with present descriptions of the elementary cross section and with what we know from other reactions about the pion FSI.

\section{DUALITY IN LEPTON SCATTERING ON THE NUCLEON}

At higher energies that correspond to invariant masses of the $W, Z N$ system $>2 \mathrm{GeV}$ the DIS channel becomes essential. A connection between the asymptotic DIS region and the resonance-dominated mass range is provided by the so-called Bloom-Gilman quark-hadron duality.

Nearly forty years ago, Bloom and Gilman found [20] that in electron scattering on protons the inclusive structure function $F_{2}$ in the resonance region oscillates around the DIS scaling curve and, after averaging, closely resembles it. Furthermore, the resonance region data "slide" along the DIS curve with increasing $Q^{2}$. If duality is understood quantitatively, there may be various applications. For example, the region of high Bjorken variable $x$ is hardly experimentally investigated, because in the DIS region it would require very high $Q^{2}$ and thus huge luminosities. If duality is satisfied with good accuracy, one would be able to use the data in the resonance region to reach high $x$ at reasonable $Q^{2}$.

The topic becomes even more interesting when turning to nuclear targets and neutrino sources. The current precision measurements of the oscillation parameters require an efficient and accurate description of the neutrino-nucleus cross sections. Of particular interest is the resonance region and the possibility of linking it with the DIS region. A hadronic description of a neutrinonucleus cross section at low $Q^{2}$ requires a good knowledge of vector and axial transition form factors for each resonance. For the majority of the resonances, these transition form factors are not well constrained. Provided that one can establish that quark-hadron duality holds with a reasonable accuracy, one could think of using the DIS results for estimating the neutrino-nucleus cross sections in the transition region.

So far, most theoretical studies of quark-hadron duality in lepton scattering were dealing with nucleon tar- gets [21, 22, 23, 24]. The DIS parts were considered as known, the structure functions in the scaling region being conventionally evaluated from leading twist (LT) parton distribution functions (PDF): for example, $F_{2}^{e N(L T)}=$ $\left(F_{2}^{e p}+F_{2}^{e n}\right) / 2=5 x / 18 \cdot(u+\bar{u}+d+\bar{d}+2 s / 5+2 \bar{s} / 5)$, $F_{2}^{v N(L T)}=\left(F_{2}^{v p}+F_{2}^{v n}\right) / 2=x(u+\bar{u}+d+\bar{d}+s+\bar{s})$. For nucleons, several parametrizations of the PDFs are generally available (from the GRV, CTEQ and MRST groups). In the region of moderate $x$, which is of interest for our duality study, they provide nearly the same results.

The studies of the resonance region differ in the way the models treat the resonant contributions and the way they extract the structure functions.

The fact, that the resonance data for increasing $Q 2$ "slide" along the DIS curve, can be observed if a few [24] or even only one [21] resonance are taken into account. The advantage of the model [24] is that the structure functions are given as simple analytical functions of the momentum transfer squared $Q^{2}$ and the energy transfer $v$, provided that the resonance form factors are known. In this work the first four resonances were considered. Generally, however, as it was argued by Close [25], inclusion of several resonances of different parities is desirable.

A quark model for resonance excitation has been applied by [22, 23] for the investigation of the duality in electro- and neutrinoproduction.

Within the GiBUU framework, as it was mentioned above, 13 resonances can be considered for both electron and neutrino reactions. The cross section is calculated numerically and the structure function $F_{2}$ is extracted from the cross section in a convenient way:

$$
\begin{gathered}
\frac{d \sigma^{N}}{d Q^{2} d v}=k_{E M, C C} \frac{\pi}{E E^{\prime}} \frac{F_{2}\left(Q^{2}, x\right)}{v} \\
{\left[1-\frac{Q^{2}}{4 E E^{\prime}}+2 \frac{Q^{2}}{4 E E^{\prime}} \frac{Q^{2} v^{2}+Q^{4}}{Q^{4}(1+R)}\right]}
\end{gathered}
$$

The ratio $R$, defined as $2 x F_{1}(1+R)=F_{2}(1+$ $\left.4 m_{N}^{2} x^{2} / Q^{2}\right)$, is the world average value

$$
\begin{array}{r}
R\left(Q^{2}, x\right)=\frac{0.0635}{\ln \left(Q^{2} / 0.04\right)}[1 .+ \\
\left.+\frac{12 . Q^{2}}{Q^{2}+1.0} \frac{0.125^{2}}{0.125^{2}+x^{2}}\right] \\
+\frac{0.5747}{Q^{2}}-\frac{0.3534}{Q^{4}+0.09}
\end{array}
$$

taken from [26]. The coefficients

$$
k_{E M}=\frac{4 \alpha_{e m}^{2} E^{\prime 2}}{Q^{4}}, \quad k_{C C}=\frac{G_{F}^{2} E^{\prime 2}}{2 \pi^{2}},
$$

are the Mott cross sections for electron and neutrino reactions. For electroproduction on an isoscalar target, $\sigma^{N}=\left(\sigma^{e p}+\sigma^{e n}\right) / 2$ is half sum of electroproduction 
cross sections on proton and neutron. For charged current neutrinoproduction, in order to eliminate the structure function $F_{3}$, one should use the linear combination of the neutrino and anti-neutrino cross sections. For an isoscalar target it is sufficient to take $\sigma^{N}=\left(\sigma^{v p}+\sigma^{\bar{v} p}+\right.$ $\left.\sigma^{v n}+\sigma^{\bar{v} n}\right) / 4$.

For a quantitative estimate of the validity of duality it is convenient to introduce the ratio of the integrals of the resonance (res) and DIS structure functions

$$
I_{i}\left(Q^{2}\right)=\frac{\int_{\xi_{\min }}^{\xi_{\max }} d \xi \mathscr{F}_{i}^{(\mathrm{res})}\left(\xi, Q^{2}\right)}{\int_{\xi_{\min }}^{\xi_{\max }} d \xi \mathscr{F}_{i}^{(\mathrm{DIS})}\left(\xi, Q_{D I S}^{2}\right)}
$$

where $\mathscr{F}_{i}$ denotes $2 x F_{1}, F_{2}$ or $x F_{3}$ (for neutrino scattering). The value $Q_{D I S}^{2}$ is taken as the actual $Q^{2}$ value for a given parametrization of DIS PDFs (for nucleon, in our case, $Q_{D I S}^{2}=10 \mathrm{GeV}$ ) or a DIS experimental data set (for nuclei). Under conditions of perfect quark-hadron duality this ratio would be 1 and independent of $Q^{2}$. Thus, the degree to which the local duality is fulfilled can be estimated from the $Q^{2}$ dependence and the deviation from 1 of the computed $I_{2}$.

The isoscalar nucleon $F_{2}^{e N}$ structure function, which includes both resonance and background contributions, is shown in Fig. 8 versus the Nachtmann variable $\xi$. Notice, that $\xi$ decreases with increasing invariant mass $W$. For a given $Q^{2}$ value, the highest peak at the larger $\xi$ value corresponds to the $\Delta$-resonance peak, and the two lower peaks at smaller values of $\xi$ correspond to the second $(1.40 \mathrm{GeV} \lesssim W \lesssim 1.56 \mathrm{GeV})$ and the third $(1.56 \mathrm{GeV} \lesssim W \lesssim 2.0 \mathrm{GeV})$ resonance regions. The general picture shows a reasonable agreement with the duality hypothesis.

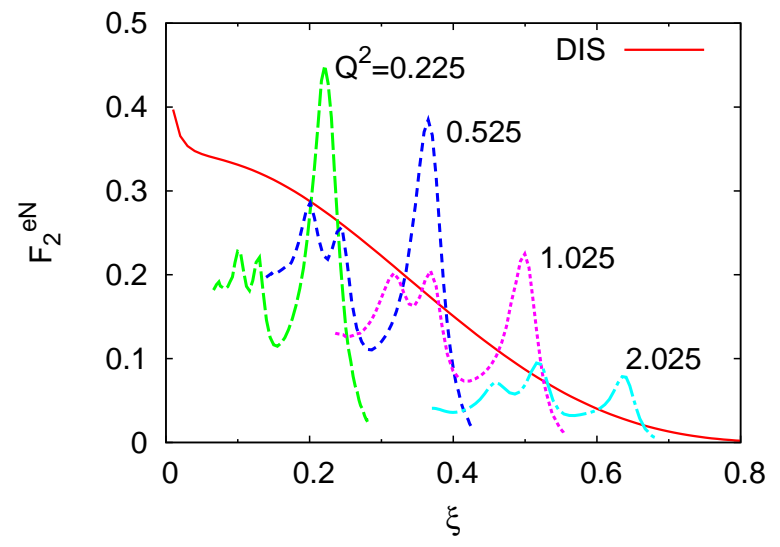

FIGURE 8. $\quad F_{2}^{e N}$ as a function of $\xi$, for $Q^{2}=0.225,0.525$, 1.025 and $2.025 \mathrm{GeV}^{2}$ (indicated on the spectra), compared with the leading twist parametrizations at $Q^{2}=10 \mathrm{GeV}^{2}$.

In Fig. 9 the ratio of the integrals $I_{2}^{e N}$, defined in (3), is shown not only for the whole structure function (resonance +1 -pion background), but also for the resonance

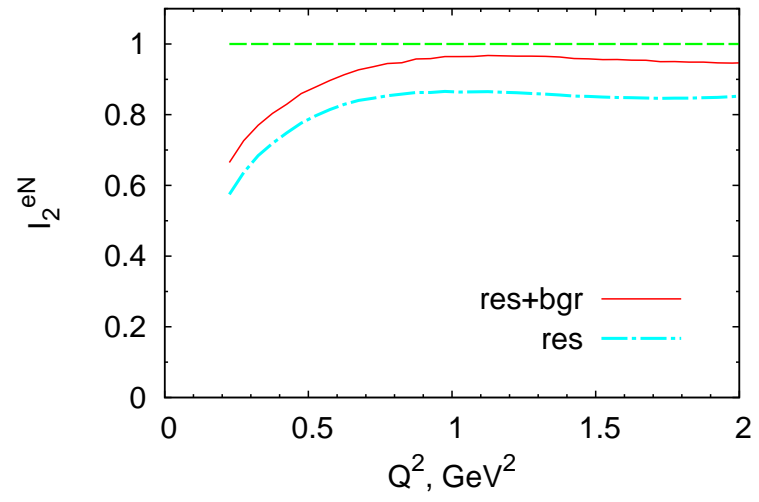

FIGURE 9. Ratio $I_{2}^{e N}$ of the integrated $F_{2}^{e N}$ in the resonance region to the leading twist functions.

contribution separately. For $Q^{2}>0.5 \mathrm{GeV}^{2}$, the ratio $I_{2}^{e N}$ for the resonance contribution only is at the level of 0.85 , which is smaller and flatter in $Q^{2}$ in comparison with the results [24, 27] of the Dortmund group resonance model. The difference is due to the different parametrization of the electromagnetic resonance form factors used in the two models. The $1 \pi$ background gives a noticeable contribution and brings the ratio up to 0.95 . The fact, that the ratio is smaller than 1 is no surprise, because additional non-resonant contributions like 2- and many-pion background are possible, but not taken into account here. They are the subject of coming investigations. Indeed, they could be obtained from requirement $I_{2}=1$.

The principal feature of neutrino reactions, stemming from fundamental isospin arguments, is that duality does not hold for proton and neutron targets separately. The interplay between the resonances of different isospins allows for duality to hold with reasonable accuracy for the average over the proton and neutron targets. We expect, that a similar picture emerges in neutrino reactions with nuclei.

For neutrinoproduction, the structure function $F_{2}^{v N}$ is shown in Fig. 10 for the resonance contribution only.

The ratio $I_{2}^{V N}$ is shown in Fig. 11 and appears to be at the level of 0.7 , which is (similar to the electron case) smaller than 0.8 , which has been calculated within the Dortmund resonance model [24, 27]. Thus, one would expect a large contribution from the background. The role of the background in the anti-neutrino channel is under investigation. 


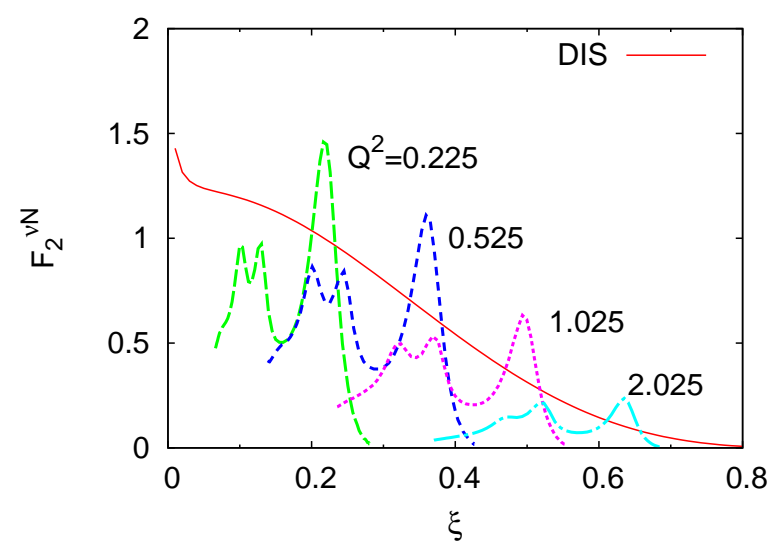

FIGURE 10. $F_{2}^{v N}$ as a function of $\xi$, for $Q^{2}=0.225,0.525$, 1.025 and $2.025 \mathrm{GeV}^{2}$ (indicated on the spectra), compared with the leading twist parametrizations at $Q^{2}=10 \mathrm{GeV}^{2}$.

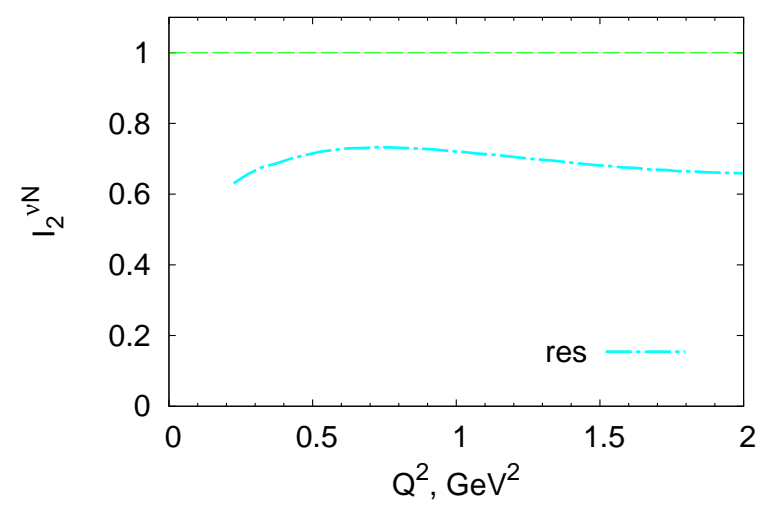

FIGURE 11. Ratio $I_{2}^{v N}$ of the integrated $F_{2}^{v N}$ in the resonance region to the leading twist functions.

\section{DUALITY IN LEPTON SCATTERING ON THE NUCLEUS}

Recent electron scattering measurements at JLab have confirmed the validity of the Bloom-Gilman duality for proton, deuterium and iron structure functions. Further experimental efforts are required for neutrino scattering. Among the upcoming neutrino experiments, Minerva[28] and SciBooNE[29] aim at measurements with carbon, iron and lead nuclei as targets.

One of the major issues for nuclear targets is the definition of the nuclear structure functions $F_{1(2,3)}^{A}$. Experimentally they are determined from the corresponding cross sections, using Eq. (2).

We follow the same procedure, using the GiBUU cross sections. So, at the first step the inclusive double differential cross section $d \sigma / d Q^{2} d v$ is calculated within the
GiBUU model. The FSI do not play any role for the inclusive cross section. The in-medium corrections are of considerable importance.

In Fig. 12, the resonance contribution to the $F_{2}^{A} / A$ structure functions for a carbon target are shown for several $Q^{2}$ values. They are compared to experimental data obtained by the BCDMS collaboration [30, 31] in muoncarbon scattering in the DIS region $\left(Q^{2} \sim 30-50 \mathrm{GeV}^{2}\right)$. They are shown as experimental points connected by smooth curves. For different $Q^{2}$ values, the experimental curves agree within $5 \%$ in most of the $\xi$ region, as expected from Bjorken scaling.

When investigating duality for a free nucleon, we took the average over free proton and neutron targets, thus considering the isoscalar structure function. Since the carbon nucleus contains an equal number of protons and neutrons, averaging over isospin is performed automatically. Due to the Fermi motion of the target nucleons, the peaks from the various resonance regions, which were clearly seen for the nucleon target, are hardly distinguishable for the carbon nucleus.

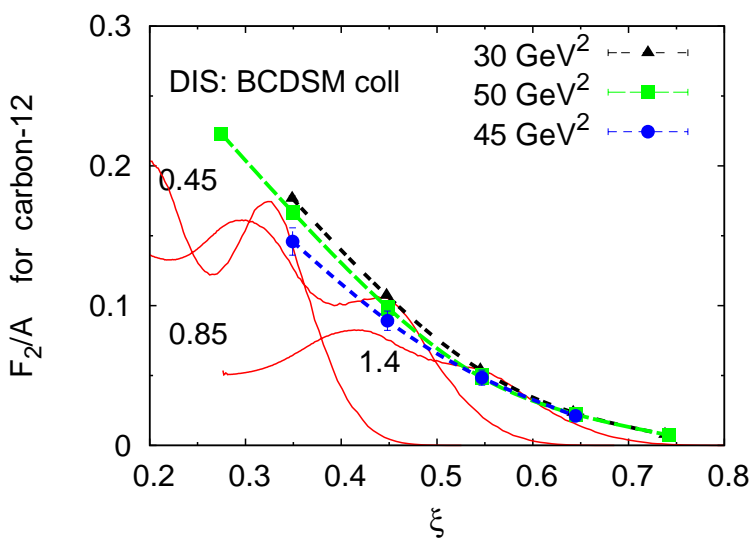

FIGURE 12. Resonance curves $F_{2}^{e^{12} C} / 12$ as a function of $\xi$, for $Q^{2}=0.45,0.85$ and $1.4 \mathrm{GeV}^{2}$ (indicated on the spectra), compared with the experimental BCDMS data [30, 31] in the DIS region at $Q_{D I S}^{2}=30,45$ and $50 \mathrm{GeV}^{2}$.

As expected from local duality, the resonance structure functions for the various $Q^{2}$ values slide along a curve, whose $\xi$ dependence is very similar to the scaling-limit DIS curve. However, for all $\xi$, the resonance curves lie below the experimental DIS data.

To quantify this underestimation, we now consider the ratio of the integrals of the resonance (res) and DIS structure functions, determined in Eq. (3). As it is explained in [32], the integration limits are to be determined in terms of the effective $\tilde{W}$ variable, experimentally (see, for example, [33]) defined as $\tilde{W}^{2}=m_{N}^{2}+2 m_{N} v-Q^{2}$. For a free nucleon $\tilde{W}$ coincides with the invariant mass $W$. For a nucleus, it differs from $W$ due to the Fermi motion of bound nucleons, but still gives a reasonable estimation 
for the invariant mass region involved in the problem.

In particular, the resonance curves presented in all figures are plotted in the region from the pion-production threshold up to $\tilde{W}=2 \mathrm{GeV}$. For a free nucleon, the threshold value for 1-pion production (and thus the threshold value of the resonance region) is $\tilde{W}_{\min }=$ $W_{\min } \approx 1.1 \mathrm{GeV}$. Bound backward-moving nucleons in a nucleus allow lower $W$ values beyond the free-nucleon limits. The threshold for the structure functions is now defined in terms of $v$ or $\tilde{W}$, rather than $W$. Hence, we consider two different cases in choosing the $\xi$ integration limits for the ratio (3). First, for a given $Q^{2}$, we choose the $\xi$ limits in the same manner as for a free nucleon:

$$
\begin{aligned}
& \xi_{\min }=\xi\left(\tilde{W}=1.6 \mathrm{GeV}, Q^{2}\right), \\
& \xi_{\max }=\xi\left(\tilde{W}=1.1 \mathrm{GeV}, Q^{2}\right) .
\end{aligned}
$$

We refer to this choice as integrating "from $1.1 \mathrm{GeV}$ ". The integration limits for the DIS curve always correspond to this choice. As a second choice, for each $Q^{2}$ we integrate the resonance curve from the threshold, that is from as low $\tilde{W}$ as is achievable for the nucleus under consideration. This corresponds to the threshold value at higher $\xi$ and is referred to as integrating "from threshold". With this choice we guarantee that the extended kinematical regions typical for resonance production from nuclei are taken into account. Since there is no natural threshold for the $\xi_{\text {min }}$, for both choices it is determined from $\tilde{W}=1.6 \mathrm{GeV}$, as defined in Eq. (4).

The results for the ratio (3) are shown in Fig. 13, The curve for the isoscalar free-nucleon case is the same as in Ref. [24] with the "GRV" parametrization for the DIS structure function. One can see that the carbon curve obtained by integrating "from threshold" lies above the one obtained by integrating "from $1.1 \mathrm{GeV}$ ", the difference increasing with $Q^{2}$. This indicates that the threshold region becomes more and more significant, as one can see from Fig. 13 Recall, that the flatter the curve is and the closer it gets to 1 , the better local duality would hold. Our calculations for carbon show that the ratio is at the same level as that for the free nucleon or even higher.

For neutrino-iron scattering, the structure functions $\mathrm{F}_{2}^{v F e}$ are shown in Fig. 14. As for the electron-carbon results of Fig. 12, the resonance structure is hardly visible. The resonance structure functions are compared to the experimental data in the DIS region obtained by the CCFR [34] and NuTeV [35] collaborations. It appears, that the resonance curves slide along the DIS curve, as one would expect from local duality, but lie well below the DIS measurements. Hence, the computed structure functions do not average to the DIS curve. The necessary condition for local duality to hold is thus not fulfilled.

As expected from local duality, the resonance structure functions for the various $Q^{2}$ values slide along a curve, whose $\xi$ dependence is very similar to the scaling-limit

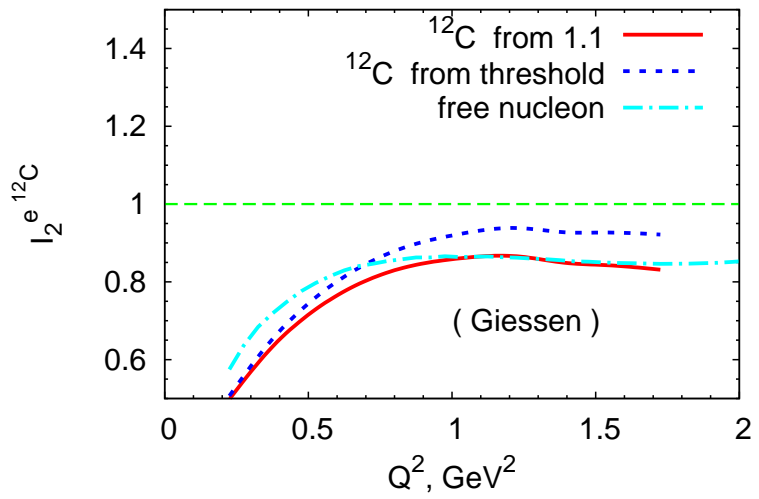

FIGURE 13. Ratio defined in Eq. (3) for the free nucleon (dash-dotted line), and ${ }^{12} C$. We consider the lower limits determined by $\tilde{W}=1.1 \mathrm{GeV}$ (solid line) and by the threshold value (dotted line).

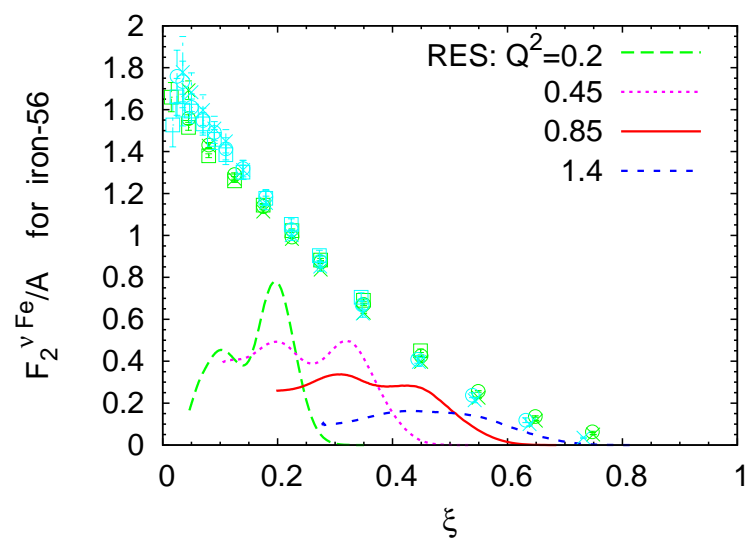

FIGURE 14. $F_{2}^{v^{56} \mathrm{Fe}} / 56$ as a function of $\xi$ for $Q^{2}=0.2,0.45$, 0.85 , and $1.4 \mathrm{GeV}^{2}$. The calculations are compared with the DIS data from CCFR and NuTeV experiments Refs. [34, 35]. The DIS data refer to measurements at $Q_{D I S}^{2}=7.94,12.6$ and $19.95 \mathrm{GeV}^{2}$.

DIS curve. However, for all $\xi$, the resonance curves lie below the experimental DIS data.

The ratio $I_{2}^{V^{56} \mathrm{Fe}}$ defined in Eq. (3) is shown in Fig. 15, The curve for the isoscalar free nucleon case is also presented for comparison. Our results show, that 1 ) this ratio is significantly smaller than 1 for all $Q^{2} ; 2$ ) it is significantly smaller than the one for the free nucleon; 3) $I_{2}$ is even lower than the corresponding ratio for electroproduction; 4) $I_{2}$ slightly decreases with $Q^{2}$.

To summarize, we find that the resonance structure functions are consistently smaller than DIS functions in the same region of the Nachtmann variable $\xi$. This is in agreement with earlier work [32], which implements elementary resonance vertices and nuclear effects differ- 


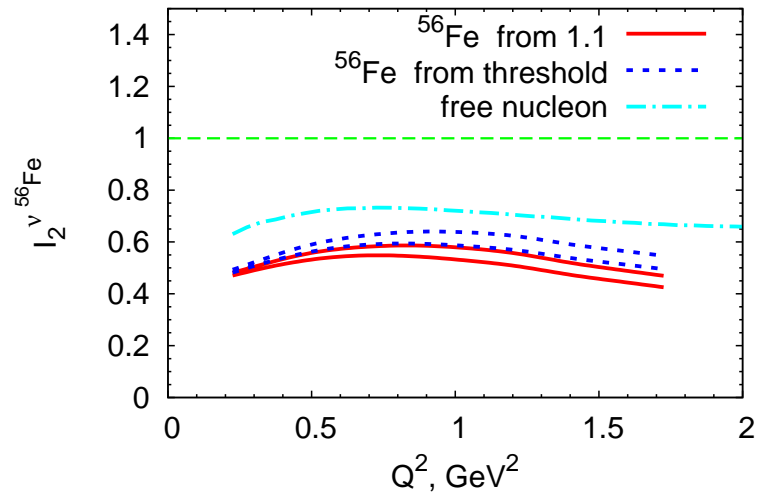

FIGURE 15. Ratio $I_{2}^{v^{56} \mathrm{Fe}}$ defined in Eq. (3) for the free nucleon (dash-dotted line) and ${ }^{56} \mathrm{Fe}$. The results are displayed for two choices of the lower limit in the integral: $\tilde{W}=1.1 \mathrm{GeV}$ (solid line) and threshold (dotted line). For each of these two choices we have used two sets of DIS data in determining the denominator of Eq. (3). These sets of DIS data are obtained at $Q_{D I S}^{2}=12.59$ and $19.95 \mathrm{GeV}^{2}$.

ently. Recall that in this analysis for nuclei we include the resonance structure functions and ignore the background ones. To estimate their contribution and compare the results with the nucleon case is one of the primary tasks of coming investigations.

\section{CONCLUSIONS}

Overall, the impact of nuclear effects impact on observables is dramatic. Final state interactions of produced pions lead to pions being absorbed in the nucleus or rescattered, thus reducing the cross section and shifting the observed pion distributions to lower values of the pion energy. Furthermore, such interactions can lead to knockout nucleons which experiments tend to identify with $\mathrm{QE}$ events. CCQE and pion production are thus closely entangled. Thus model-independent data are needed for a meaningful comparison of theory with current experiments. Initial state interactions reduce the cross sections and structure functions in the resonance region and change the form of the distributions, dissolving the peaks of individual resonances. The Bloom-Gilman duality seems to be violated for nuclear targets, possibly indicating a major change of background amplitudes in the medium.

\section{ACKNOWLEDGMENTS}

This work has been supported by the Deutsche Forschungsgemeinschaft.

\section{REFERENCES}

1. T. Leitner, O. Buss, L. Alvarez-Ruso and U. Mosel, Phys. Rev. C79, 034601 (2009), arXiv:0812.0587.

2. MAID, http://www.kph.uni-mainz.de/MAID

3. L. Tiator and S. Kamalov, arXiv:nucl-th/0603012

4. D. Drechsel, S. S. Kamalov and L. Tiator, Eur. Phys. J. A34, 69 (2007), arXiv:0710.0306.

5. S. J. Barish et al., Phys. Rev. D19, 2521 (1979).

6. G. M. Radecky et al., Phys. Rev. D25, 1161 (1982).

7. T. Kitagaki et al., Phys. Rev. D34, 2554 (1986).

8. GiBUU, http://gibuu.physik.uni-giessen.de/GiBUU

9. B. Krusche et al., Eur. Phys. J. A22, 277 (2004), arXiv:nucl-ex/0406002.

10. O. Buss, L. Alvarez-Ruso, P. Muehlich and U. Mosel, Eur. Phys. J. A29 (2), 189 (2006), arXiv:nucl-th/0603003

11. O. Buss, L. Alvarez-Ruso, A. B. Larionov and U. Mosel, Phys. Rev. C 74, 044610 (2006), arXiv:nucl-th/0607016

12. MiniBooNE, T. Katori, arXiv:0909.1996 [hep-ex].

13. MiniBooNE, A. A. Aguilar-Arevalo et al., Phys. Rev. Lett. 103, 081801 (2009), arXiv:0904.3159.

14. T. Leitner, O. Buss, U. Mosel and L. Alvarez-Ruso, arXiv:0909.0838

15. M. S. Athar, S. Chauhan and S. K. Singh, arXiv:0908.1442

16. MiniBooNE, A. A. Aguilar-Arevalo et al., Phys. Rev. D79, 072002 (2009), arXiv:0806.1449.

17. T. Leitner, O. Buss, U. Mosel and L. Alvarez-Ruso, Phys. Rev. C79, 038501 (2009), arXiv:0812.1787.

18. C. Anderson, Neutral current $1 \pi^{0}$ production at MiniBooNE, talk given at NUINT 09, May 17-22, 2009, Sitges, Spain, available online at http://nuint09.ifae.es/Agenda.html

19. K2K, S. Nakayama et al., Phys. Lett. B619, 255 (2005), arXiv:hep-ex/0408134.

20. E. D. Bloom and F. J. Gilman, Phys. Rev. Lett. 25, 1140 (1970).

21. K. Matsui, T. Sato and T. S. H. Lee, Phys. Rev. C72, 025204 (2005), arXiv:nucl-th/0504051

22. K. M. Graczyk, C. Juszczak and J. T. Sobczyk, Nucl. Phys. A781, 227 (2007), arXiv:hep-ph/0512015

23. K. M. Graczyk, arXiv:0910.2355 [hep-ph].

24. O. Lalakulich, W. Melnitchouk and E. A. Paschos, Phys. Rev. C75, 015202 (2007), arXiv:hep-ph/0608058.

25. F. E. Close and W. Melnitchouk, Phys. Rev. C68, 035210 (2003), arXiv:hep-ph/0302013

26. L. W. Whitlow, E. M. Riordan, S. Dasu, S. Rock and A. Bodek, Phys. Lett. B282, 475 (1992).

27. O. Lalakulich et al., AIP Conf. Proc. 967, 243 (2007).

28. Minerva, http://minerva.fnal.gov

29. SciBooNE, http://www-sciboone.fnal.gov/

30. D. Bollini et al., Phys. Lett. B104, 403 (1981).

31. BCDMS, A. C. Benvenuti et al., Phys. Lett. B195, 91 (1987).

32. O. Lalakulich, N. Jachowicz, C. Praet and J. Ryckebusch, Phys. Rev. C79, 015206 (2009), arXiv:0808.0085

33. R. M. Sealock et al., Phys. Rev. Lett. 62, 1350 (1989).

34. W. G. Seligman et al., Phys. Rev. Lett. 79, 1213 (1997).

35. NuTeV, M. Tzanov et al., Phys. Rev. D74, 012008 (2006), arXiv:hep-ex/0509010. 